\title{
Circulating neutrophil-to-lymphocyte ratio at admission predicts the long-term outcome in acute traumatic cervical spinal cord injury patients
}

Jian-Lan Zhao ${ }^{1,2,3,4 \dagger}$, Song-Tao Lai ${ }^{5 \dagger}$, Zhuo-Ying Du ${ }^{1,2,3,4 \dagger}$, Jian Xu' ${ }^{6}$, Yi-Rui Sun ${ }^{1,2,3,4}$, Qiang Yuan ${ }^{1,2,3,4}$, Xing Wu ${ }^{1,2,3,4}$, Zhi-Qi Li $i^{1,2,3,4}$, Jin $\mathrm{Hu}^{1,2,3,4^{*}}$ and Rong Xie $\mathrm{Xi}^{1,2,3,4^{*}}$

\begin{abstract}
Background: The prognostic value of Neutrophil-to-Lymphocyte Ratio (NLR) for the outcome of acute cervical traumatic spinal cord injury ( $\mathrm{tSCl}$ ) patients has rarely been studied by now throughout the world.

Methods: We performed a single-center retrospective cohort study to evaluate the prognostic value of NLR from peripheral whole blood count in patients with acute cervical $\mathrm{tSCl}$. Patients within $6 \mathrm{~h}$ of acute cervical $\mathrm{tSCl}$ treated between Dec 2008 and May 2018 in Huashan Hospital of Fudan University were enrolled. Outcomes of patients with $\mathrm{tSCl}$ were assessed using American spinal injury association Impairment Scale (AIS). 6-month outcomes were dichotomized into poor outcome group (AIS A to C) and good outcome group (AIS D and E). Uni- and multivariate analyses were performed to assess the independent predictors of 6-month outcome. Two prediction models based on admission characteristics were built to evaluate the prognostic value of NLR. The discriminative ability of predictive models was evaluated using the area under the curve (AUC).

Results: A total of 377 patients were identified from our single center in China PR. Multivariate analysis showed that age, AIS grade at admission, NLR $(p<0.001)$ and coagulopathy $(p=0.003)$ were independent predictors of the 6 -months outcome for acute cervical tSCl patients. The model combing NLR and standard variables (AUC = 0.944; $95 \% \mathrm{Cl}, 0.923-0.964)$ showed a more favorable prognostic value than that without NLR (AUC $=0.841 ; 95 \% \mathrm{Cl}, 0.798-$ 0.885) in terms of 6-month outcome.

Conclusions: NLR is firstly identified as an independent predictor of the 6-month outcome in acute cervical tSCl patients worldwide. The prognostic value of NLR is favorable, and a high NLR is associated with poor outcome in patients with acute cervical tSCl.
\end{abstract}

Keywords: Acute traumatic cervical spinal cord injury, Neutrophil-to-lymphocyte ratio, Prediction model, Prognostic value, 6-months outcome

\footnotetext{
*Correspondence: 286644104@qq.com; hujin_dana@126.cn;

rongxie@fudan.edu.cn

†Jian-Lan Zhao, Song-Tao Lai and Zhuo-Ying Du contributed equally to this

work.

${ }^{1}$ Department of Neurosurgery, Huashan Hospital, Shanghai Medical College, Fudan University, 12 Wulumuqi Road (M), Shanghai 200040, P.R. China

Full list of author information is available at the end of the article
}

C C The Author(s). 2020 Open Access This article is licensed under a Creative Commons Attribution 4.0 International License, which permits use, sharing, adaptation, distribution and reproduction in any medium or format, as long as you give appropriate credit to the original author(s) and the source, provide a link to the Creative Commons licence, and indicate if changes were made. The images or other third party material in this article are included in the article's Creative Commons licence, unless indicated otherwise in a credit line to the material. If material is not included in the article's Creative Commons licence and your intended use is not permitted by statutory regulation or exceeds the permitted use, you will need to obtain permission directly from the copyright holder. To view a copy of this licence, visit http://creativecommons.org/licenses/by/4.0/. The Creative Commons Public Domain Dedication waiver (http://creativecommons.org/publicdomain/zero/1.0/) applies to the data made available in this article, unless otherwise stated in a credit line to the data. 


\section{Background}

Traumatic spinal cord injury (tSCI), especially the cervical spinal cord, is one of the most devastating form of trauma because of its high morbidity rate and enormous financial and social burden. The prevalence of tSCI is approximately 750 per million worldwide with a trend of rising annual incidence [1]. Similar to traumatic brain injury (TBI), the primary injury of spinal cord is induced by trauma impact, while the secondary injury is triggered by multiple factors during different time courses after injury, among which the inflammatory response plays a vital role $[2,3]$.

It was recognized that either primary or secondary injury was associated with the outcome of tSCI patients was considered to be $[4,5]$, and several standard prognostic factors at admission, including age, blood cells counts, coagulation status, Charleson Co-morbidity Index (CCI), American spinal injury association Impairment Scale (AIS) grades, and the initial Glasgow Coma Scale (GCS) score, were identified to be associated with the outcome of tSCI patients [6, 7]; however, their prognostic values were limited even a variety of predictive models were built. Thus, to develop a model with higher prognostic value is justified.

As mentioned previously, inflammation responses, which are considered to contribute to the secondary $\mathrm{tSCI}$, are partly activated by purinergic signaling in the acute phase [8]. After focal tSCI, circulating neutrophils can be observed to be recruited to the site of injury within $1 \mathrm{~h}$, which is considered as an indicator of acute inflammation [9]. It was reported that high neutrophils and low lymphocytes level in the peripheral blood at admission were independently associated with poor outcome of intracerebral hemorrhage $(\mathrm{ICH})$ patients, and the neutrophil-to-lymphocyte ratio (NLR) was readily available as an outcome predictor $[10,11]$. Nevertheless, the prognostic value of NLR for the outcome of tSCI patients has rarely been studied. Moreover, whether combining NLR with standard independent predictors would improve prognostic is still unknown.

Our study evaluated the prognostic value of NLR and tested its predictive power in prediction models in terms of the 6-months outcome for cervical tSCI patients.

\section{Methods}

\section{Study design and participants}

We performed a retrospective, observational cohort study in Huashan Hospital of Fudan University. This study was approved by the Institutional Review Board of Huashan Hospital of Fudan University. Between Dec. 2008 and May. 2018, patients with acute tSCI who were admitted to the Department of the Neurosurgery were recruited. Written informed consent was obtained from all individual participants.
Inclusion criteria were set as follow: 1) patients with a diagnosis of traumatic spinal cord injury confirmed by computed tomography $(\mathrm{CT}) ; 2$ ) more than 14 years of age; 3 ) within $6 \mathrm{~h}$ after injury; 4) initial GCS $>13$; 5) initial AIS grade A to D and 6) C1 to T1segment spinal injury. Exclusion criteria included: 1) patients with traumatic injury to a body region other than the cervical spinal cord with an Abbreviated Injury Severity (AIS) score $\geq 3$; 2) a penetrating neck injury; 3) pregnancy; 4) pre-injury major neurologic deficits or disease (i.e. ischemic stroke, Parkinson's Disease) and 5) injury related medical treatments, including methylprednisolone, spinal surgeries and et al., in other hospitals or medical centers before admission. All patients were evaluated and treated by full-time neurosurgeons with specific training in critical care.

\section{Blood cells count and demographic data}

At admission, blood samples of included patients were collected and analyzed by the Central Clinical Chemistry Laboratory of Huashan Hospital. White blood cells (WBC), neutrophil ratio and lymphocyte ratio were routinely performed in all patients within $6 \mathrm{~h}$ of injury. Neurologic examinations were performed according to the standards established by the American Spinal Injury Association (ASIA).

Clinical and demographic characteristics, including age, gender, types of injury, Charleson Co-morbidity Index (CCI), initial Glasgow Coma Scale (GCS) score, and the overall ASIA Impairment Scale (AIS) grade were recorded for all patients. Injury types were assessed by using initial CT scans on admission.

Coagulopathy was defined as our previous studies [12, 13]. Briefly, platelet counts (PLT) $<100 \times 10^{9} / \mathrm{L}$, international normalized ratio (INR) $>1.25$, prothrombin time $(\mathrm{PT})>14 \mathrm{~s}$, and activated partial thromboplastin time $($ APTT $)>36 \mathrm{~s}$.

\section{Outcome assessments}

The primary outcome was ordinal change in AIS grade at 6 months after injury, which was in accordance to the recommendations by the National Acute Spinal Cord Injury Study (NASCIS). Moreover, several notable natural history studies and clinical trials such as Sygen trial has already demonstrated that the vast majority of patients showed signs of neurological recovery during this period. We carefully assessed the 6-months outcome of SCI patients via outpatient interviews or over the phone after discharge. The outcome of included patients was dichotomized into poor (AIS A to C) and good outcome (AIS D and E) $[14,15]$. Relevant postoperative complications were also recorded. 


\section{Predictive models and statistical analysis}

The prognostic models derived from the data of included patients. Two models for 6-months outcome were developed based on admission characteristics: model A included standard predictors such as age, gender, mechanism of injury, AIS grades at admission, and types of injury; model B included the results of WBC counts, neutrophil ratio, lymphocyte ratio and NLR in addition to the predictors in model $\mathrm{A}$.

Continuous variables/predictors were expressed as means \pm standard deviation (SD) or medians (interquartile range) and categorical variables as percentages. The univariate analyses of categorical data were performed using the chi-squared test. Equality of variance was assessed using Levene's test. Normally distributed variables were compared using Student's t-tests or one-way ANOVA, whereas non-normally distributed variables were compared using the Kruskal-Wallis or MannWhitney U-tests.

Following the univariate analyses, a forward stepwise logistic regression analysis of the 6-months outcome was used to develop the prediction models and adjust for multiple predictors of 6-months outcome. By constructing receiver operative curve (ROC) and calculating the area under the curve (AUC), we evaluated the specificity and sensitivity of the two models, and their discriminative power. All statistical tests were two-tailed and $p$ values $<0.05$ were considered statistically significant. Statistical analysis was performed using SPSS 23.0 (IBM, USA) and MedCalc statistical software (version 15.2.2, MedCalc Software bvba, Ostend, Belgium).

\section{Results}

A total of 377 cervical tSCI patients were included in our study. The flowchart of participants' selection is shown as Fig. 1. Favorable outcome was confirmed in $205(54.4 \%)$ patients among the 377 included patients.

The univariate analysis revealed that age, baseline AIS grade, and coagulopathy were significantly related with the 6-months outcome $(p<0.001)$. Meanwhile, WBC counts, neutrophil ratio, lymphocyte ratio and NLR also showed close correlation with the outcome of cervical tSCI patients $(\mathrm{p}<0.001)$. Patients with poor outcome had a significantly higher WBC counts, neutrophil ratio, NLR and lower lymphocyte ratio than those with good outcome (Table 1).

The degree of neurologic improvement was measured by changes in AIS grade from admission to 6 months follow-up (Table 2). AIS grade improvements of the 377 included patients were as follows: $159(42.2 \%)$ had no improvement, 148 (39.2\%) had a 1 grade improvement, $55(14.6 \%)$ had a 2 grades improvement, $9(2.4 \%)$ had a 3 grades improvement and $6(1.6 \%)$ had a 1 grade worsening.

To analyze and adjust for multiple predictors, we further performed a forward stepwise logistic regression analysis. After adjustment, NLR remained a statistically significant prognostic factor of 6-months outcome of cervical tSCI patients (OR, 0.93; 95\% CI, $0.87-0.98 ; p<0.001$ ), while WBC counts, neutrophil ratio and lymphocyte showed no significant correlation with patients' 6-months outcome (Table 3). Other independent prognostic factors included age, baseline AIS grade $(\mathrm{p}<0.001)$ and coagulopathy $(p=$ 0.003) (Table 4).

Lastly, we developed two predictive models for the 6months outcome. To assess their discriminative ability, we constructed ROC and calculated AUC (Fig. 2). It is indicated that the predictive model with NLR $(\mathrm{AUC}=$ 0.944; 95\% CI, 0.923-0.964) showed a more favorable discrimination than that of the model without NLR (AUC $=0.841 ; \quad 95 \% \quad$ CI, 0.798-0.885). Thus, the

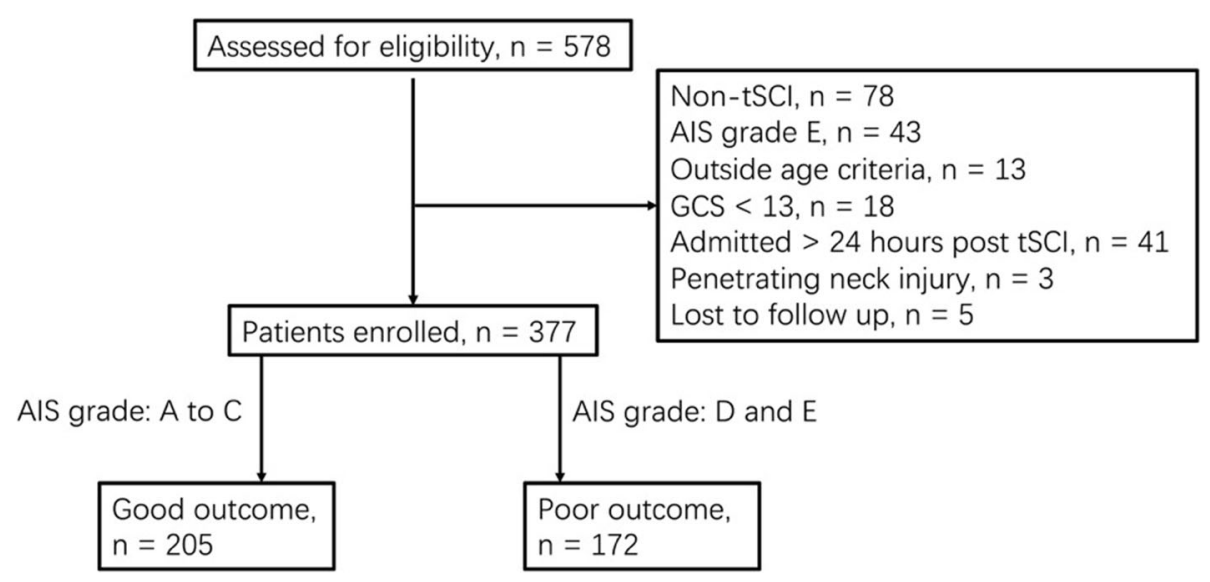

Fig. 1 Flowchart of patients' selection 
Table 1 Baseline Characteristics According to the 6-months Outcome

\begin{tabular}{|c|c|c|c|c|}
\hline & Full cohort & Poor outcome (AIS A to C) & Good outcome (AIS D to E) & $P$ value \\
\hline N & 377 & 172 & 205 & \\
\hline Age (yrs) (mean $\pm S D)$ & $46.05 \pm 17.93$ & $52.91 \pm 13.61$ & $44.61 \pm 16.34$ & $<0.001$ \\
\hline Male (N, \%) & $212(56.2)$ & $102(59.3)$ & $110(48.9)$ & 0.298 \\
\hline Mechanism of injury (n, \%) & & & & 0.762 \\
\hline Motor vehicle accident & $87(23.1)$ & $38(22.1)$ & 49 (23.9) & \\
\hline Fall & $99(26.3)$ & $51(29.7)$ & $48(23.4)$ & \\
\hline Stumble & $102(27.1)$ & $49(28.5)$ & $53(25.8)$ & \\
\hline Blow to spine & $68(18.0)$ & $31(18.1)$ & $37(18.1)$ & \\
\hline Others & $21(5.5)$ & $8(4.6)$ & $13(6.3)$ & \\
\hline GCS at admission & $14.8 \pm 0.4$ & $14.7 \pm 0.4$ & $14.8 \pm 0.3$ & 0.562 \\
\hline \multicolumn{5}{|l|}{ Baseline AIS grade $(n, \%)$} \\
\hline A & $129(34.2)$ & $80(46.5)$ & $49(23.9)$ & $<0.001$ \\
\hline B & $61(16.2)$ & $48(27.9)$ & $13(6.3)$ & $<0.001$ \\
\hline C & $72(19.1)$ & $51(29.6)$ & $21(10.2)$ & $<0.001$ \\
\hline $\mathrm{D}$ & $115(30.5)$ & $3(1.7)$ & $112(54.6)$ & $<0.001$ \\
\hline Coagulopathy (n, \%) & $87(23.1)$ & $59(34.3)$ & $28(13.7)$ & $<0.001$ \\
\hline Charleson Co-morbidity Index > 1 (n, \%) & 79 (20.9) & $41(20.9)$ & $38(20.7)$ & 0.253 \\
\hline WBCs, $\left(\times 10^{9} / \mathrm{L}\right)$ & $14.68 \pm 5.19$ & $19.01 \pm 6.32$ & $13.18 \pm 4.59$ & $<0.001$ \\
\hline Neutrophil ratio & $0.87 \pm 0.09$ & $0.89 \pm 0.09$ & $0.81 \pm 0.05$ & $<0.001$ \\
\hline Lymphocyte ratio & $0.13 \pm 0.06$ & $0.09 \pm 0.03$ & $0.12 \pm 0.07$ & $<0.001$ \\
\hline NLR & $13.28 \pm 11.46$ & $25.73 \pm 11.36$ & $7.98 \pm 7.31$ & $<0.001$ \\
\hline
\end{tabular}

Data are given as mean \pm SD, or $n(\%)$ unless otherwise noted

prognostic ability of NLR to the 6-months outcome of cervical tSCI patients is favorable.

\section{Discussion}

The main finding of our study was that cervical tSCI patients with lower 6-months AIS grades (A to C) showed significantly higher circulating NLR than those with higher AIS grades ( $D$ or E), and the level of circulating NLR was an independent prognostic factor of 6-months outcome of cervical tSCI patients. Moreover, by calculating $\mathrm{AUC}$, we found that the predictive model, combining age, gender, mechanism of injury, coagulation status and NLR, showed good discrimination than models based on solely on standard predictors. Thus, it is inferred that the predictive power of standard model can be improved

Table 2 Ordinal changes in AIS grade from admission to 6 months follow-up

\begin{tabular}{lllllll}
\hline AlS grade at admission & A & B & C & D & E & Total \\
\hline A & 67 & 34 & 22 & 6 & 0 & 129 \\
B & 2 & 13 & 19 & 24 & 3 & 61 \\
C & 0 & 1 & 14 & 48 & 9 & 72 \\
D & 0 & 0 & 3 & 65 & 47 & 115 \\
\hline
\end{tabular}

AIS American spinal injury association Impairment Scale (AIS) by circulating NLR in patients with acute cervical spinal cord injury.

For that outcome prediction at admission is important for cervical tSCI patients, NLR has rarely been studied as a prognostic factor for cervical tSCI patients. Predictive models can promote quality control through the standardization of the parameters for patient assessment which can be compared across physicians and institutions [16]. The prognostic value of predictors is determined by their reliability on assessment, the prevalence of abnormalities, and the strength of the prognostic effect [17]. Predictors we used in this study, including age, gender, coagulation status and components of circulating blood sample, can be readily obtained on admission,

Table 3 Associations of Leukocyte Counts and Neutrophil-toLymphocyte Ratio (NLR) with the 6-months outcome of tSCl patients

\begin{tabular}{|c|c|c|c|c|}
\hline \multirow{2}{*}{$\begin{array}{l}\text { Independent } \\
\text { Variable }\end{array}$} & \multicolumn{2}{|l|}{ Unadjusted } & \multicolumn{2}{|l|}{ Adjusted } \\
\hline & OR $(95 \% \mathrm{Cl})$ & P Value & OR $(95 \% \mathrm{Cl})$ & P Value \\
\hline WBCs $\left(\times 1,000 / \mathrm{mm}^{3}\right)$ & $0.82(0.77-0.83)$ & $<0.001$ & $1.02(0.94-1.07)$ & 0.156 \\
\hline Neutrophil ratio & $0.76(0.72-0.81)$ & $<0.001$ & $0.91(0.82-1.01)$ & 0.168 \\
\hline Lymphocyte ratio & $0.82(0.74-0.93)$ & $<0.001$ & $1.11(1.04-1.22)$ & 0.103 \\
\hline NLR & $0.83(0.77-0.90)$ & $<0.001$ & $0.93(0.87-0.98)$ & $<0.001$ \\
\hline
\end{tabular}


Table 4 Multivariate logistic regression analysis predicting the 6-months outcome

\begin{tabular}{lll}
\hline Independent variable & Adjusted OR (95\% Cl) & p value \\
\hline Age & $0.91(0.86-0.97)$ & $<0.001$ \\
GCS at admission & $1.18(0.93-1.34)$ & 0.713 \\
Charleson Co-morbidity Index $>1$ & $1.02(0.88-1.16)$ & 0.328 \\
Coagulopathy & $0.84(0.64-0.97)$ & 0.003 \\
Baseline AIS grade & & \\
A & 1 & - \\
B & $0.51(0.39-0.81)$ & $<0.001$ \\
C & $0.59(0.28-0.72)$ & $<0.001$ \\
D & $0.62(0.41-0.93)$ & $<0.001$ \\
Neutrophil-to-lymphocyte ratio & $0.93(0.87-0.98)$ & $<0.001$ \\
\hline
\end{tabular}

$\mathrm{Cl}$ confidence interval. OR odds ratio. The reference category value was transformed from 0 to 1 to compare subgroups

and their prognostic value had previously been confirmed either in tSCI or TBI patients [6, 17-19]. The counts of WBCs and its components, including neutrophils ratio, lymphocytes ratio and NLR, are readily available lab tests with standardized results, although rarely studied, it is reasonable to include these results into this prognostic model.

Favorable prognostic value of circulating NLR at admission had been reported in ICH and TBI patients $[11,20]$. It is expected that the prognostic value of NLR would be favorable in predicting the outcome of patients with cervical tSCI which shares the similar secondary injury mechanism as TBI. Both Uni- and multi-variate analysis showed significant correlation between NLR and patient outcome, and the prognostic effect remained substantial following adjusted analysis, which suggesting NLR is of considerable prognostic relevance in cervical tSCI patients.

As the importance of circulating blood components in predicting outcome is increasingly recognized, a variety of parameters, as monocyte to HDL cholesterol ratio, [21] and LDL-C/HDL-C ratio [22] have been studied and applied in predicting the outcome of patients with acute ischemic or hemorrhagic cerebral diseases. NLR, which conveys crucial information about the complex inflammatory activity in vascular bed, is an established marker of systemic inflammation and is easily calculated [23]. In ICH patients, a high NLR at admission was associated with poor outcome, but its underlying mechanism remained unclear [11]. In the current study, cervical tSCI patients who had a higher NLR were more likely to have a poor outcome measured by AIS grade, which is consistent with results from ICH or TBI patients [12]. In addition, the level of NLR is similar with that in TBI patients, but much higher than the level in $\mathrm{ICH}$ patients. It is assumed that unlike the primary injury in $\mathrm{ICH}$, which

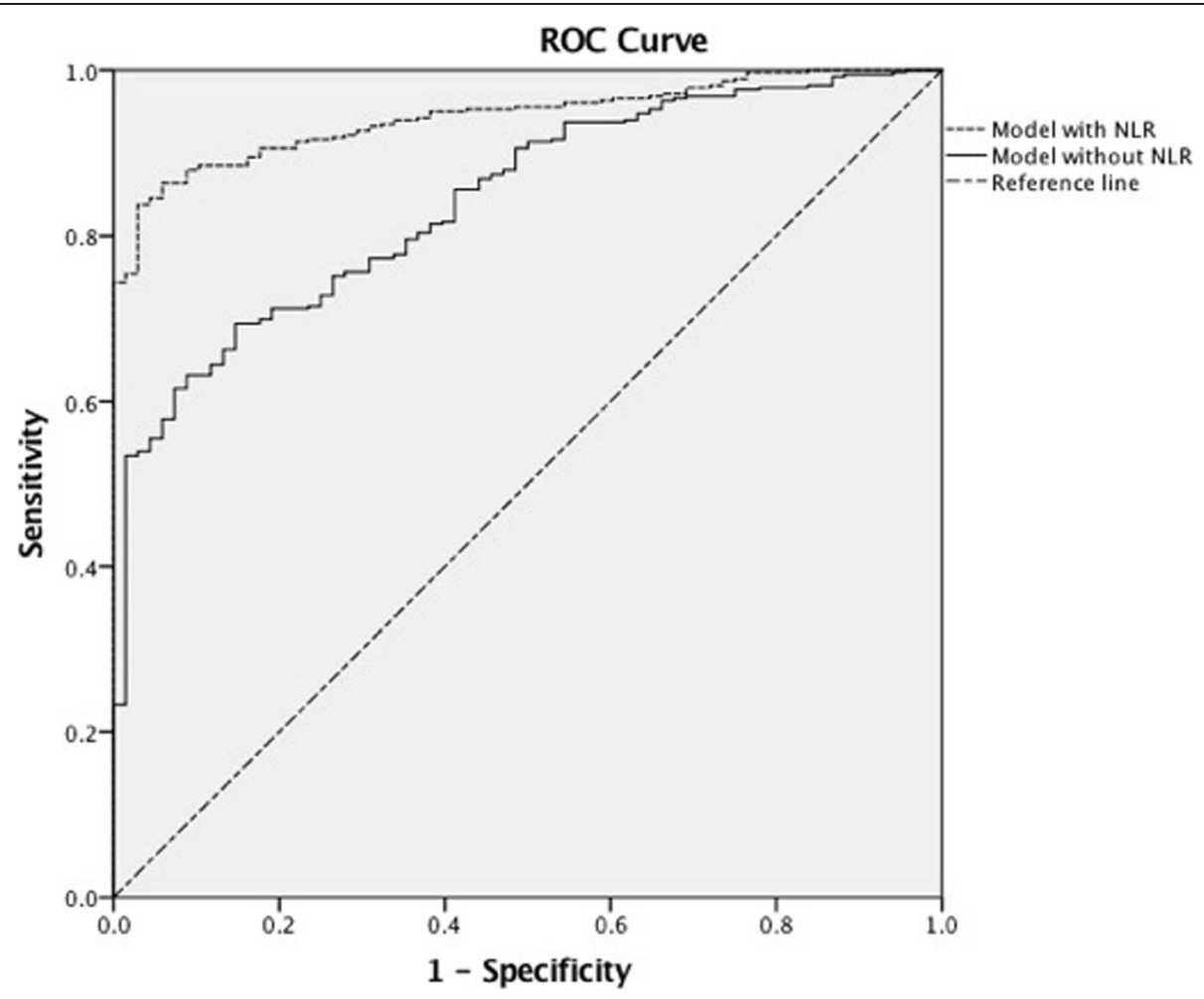

Fig. 2 Receiver operative curve (ROC) of the two predictive models. The model with Neutrophil-to-Lymphocyte Ratio (NLR) had a larger are under the curve $(A \cup C)$. It is indicated that the discrimination of the model with NLR is more favorable than the other two models 
is mainly induced by focal hematoma compression, primary injury in $\mathrm{tSCI}$ is more massive and diffuse, including the spinal cord laceration and contusion, resulting more severe acute inflammatory responses. Being considered as an indicator of severity level of acute inflammatory responses, the neutrophil counts will increase more dramatically compared with other leukocytes after spinal cord injury. Increasing of lymphocyte indicated the occurrence of chronic inflammations or a virus infection, in the other word, after acute tSCI, lymphocyte counts may will not dramatically increase. As a result, NLR level increases at early stage after spinal cord injury and is much higher than that in $\mathrm{ICH}$ patients.

It was recognized that secondary inflammatory injury was significantly associated with poor outcome in traumatic central nervous system (CNS) injury patients [3, $24,25]$. And its potential mechanism may be evolved with damage-associated molecular patterns (DAMP) released after CNS injury, and inflammatory response may initially be triggered by these DAMP [26, 27]. Moreover, significant leukocytes infiltration can be observed after CNS injury, and thus, focal inflammatory response can be aggravated, and finally, worsened inflammatory related CNS injury [28]. In the case of acute tSCI, the neutrophils are actively recruited around injury site, and contribute to the cellular injury and disruption of viable spinal cord tissues. Dramatic increase of neutrophils is seen as early as within the first hour of injury, while that of lymphocyte shows no significant changes.

Our study had several limitations. Firstly, the time course of our study was relatively long, thus the level of emergency may be different. Secondly, we included only cervical $\mathrm{tSCI}$, thus a further study is required to clarify such assumption in patients with lumbar or thoracic injury. Thirdly, the predictive ability of inflammation markers, such as C-reactive protein et al., might also play a role in outcome prediction, which would be investigated in our future studies. Last but not least, a prospective multi-center study is justified to further elucidate the relationship of NLR and 6-months outcome of patients with $\mathrm{tSCI}$, moreover, a survival statistic is also required,

\section{Conclusion}

NLR is firstly identified as an independent predictor of the 6-month outcome in acute cervical tSCI patients worldwide. The prognostic value of NLR is favorable, and a high NLR is associated with poor outcome in patients with acute cervical tSCI.

\footnotetext{
Abbreviations

AIS: American spinal injury association Impairment Scale; APT: Activated partial thromboplastin time; AUC: Area under the curve; CCl: Charleson Comorbidity Index; CT: Computed tomography; GCS: Glasgow Coma Scale; INR: International normalized ratio; NLR: Neutrophil-to-Lymphocyte Ratio; OR: Odds ratios; PLT: Platelet counts; tSCl: traumatic spinal cord injury; WBC: White blood cell
}

\section{Acknowledgments}

We are grateful to Professor Liang-Fu Zhou for his support on this study. We would also like to thank all physicians who participated in this study.

\section{Data access of the study}

The datasets used and/or analysed during the current study available from the corresponding author on reasonable request.

\section{Authors' contributions}

JLZ: study design, data collection and manuscript writing; STL: data collection and analyzation, manuscript revision; ZYD: data collection and analyzation; JX: data collection and analyzation; YRS: data collection and manuscript revision; QY: data collection and analyzation; XW: data collection and analyzation; ZQL: data collection and analyzation; JH: study design, data analyzation and manuscript revision; RX: study design, data analyzation and manuscript final revision. The author(s) read and approved the final manuscript.

\section{Funding}

This work was supported by the National Natural Science Foundation of China (81471241, 81271375, 81701206, 81671200, 81571111, 81870909, 81870968 and 81171133), Shanghai Municipal Science and Technology Commission project (16411955300, 18441903300 and 18695841300),

Shanghai Municipal Commission of Health and Family Planning project (201840063, 201801075), The Major and Develop Project of Chinese Ministry of Science and Technology (2017ZX09304005), the Public Medical Artificial Intelligence Training Platform (XX-RGZN-01-17-4684), and Shanghai Minhang Natural Science Foundation (2018MHZ001).

\section{Ethics approval and consent to participate}

This study was approved by the Institutional Review Board of Huashan Hospital of Fudan University. Informed consent was obtained from all individual participants.

\section{Consent for publication}

Not Applicable.

\section{Competing interests}

Jian-Lan Zhao, Song-Tao Lai, Zhuo-Ying Du, Yi-Rui Sun, Qiang Yuan, Xing Wu, Zhi-Qi Li, Jin Hu and Rong Xie declared that they have no conflict of interest.

\section{Author details}

'Department of Neurosurgery, Huashan Hospital, Shanghai Medical College, Fudan University, 12 Wulumuqi Road (M), Shanghai 200040, P.R. China. ${ }^{2}$ Neurosurgical Institute of Fudan University, 12 Wulumuqi Road (M), Shanghai 200040, P.R. China. ${ }^{3}$ Shanghai Clinical Medical Center of Neurosurgery, 12 Wulumuqi Road (M), Shanghai 200040, P.R. China. ${ }^{4}$ Shanghai Key laboratory of Brain Function and Restoration and Neural Regeneration, 12 Wulumuqi Road (M), Shanghai 200040, P.R. China. ${ }^{5}$ Department of Radiation Oncology, Fudan University Shanghai Cancer Center, Department of Oncology, Shanghai Medical College, Fudan University, Shanghai 200032, China. ${ }^{6}$ Department of General Surgery, the Seventh Affiliated Hospital, SUN Yat-sen University, Shenzhen 518000, China.

Received: 9 December 2019 Accepted: 3 August 2020

Published online: 15 August 2020

References

1. van den Berg ME, Castellote JM, Mahillo-Fernandez I, de Pedro-Cuesta J. Incidence of spinal cord injury worldwide: a systematic review. Neuroepidemiology. 2010;34(3):184-92 discussion 92.

2. Zhang N, Yin Y, Xu SJ, Wu YP, Chen WS. Inflammation \& apoptosis in spinal cord injury. Indian J Med Res. 2012;135:287-96.

3. Faden Al, Wu J, Stoica BA, Loane DJ. Progressive inflammation-mediated neurodegeneration after traumatic brain or spinal cord injury. $\mathrm{Br} J$ Pharmacol. 2016;173(4):681-91.

4. Hasler RM, Exadaktylos AK, Bouamra O, Benneker LM, Clancy M, Sieber R, et al. Epidemiology and predictors of spinal injury in adult major trauma patients: European cohort study. Eur Spine J. 2011;20(12):2174-80. 
5. Qiu Z, Wang F, Hong Y, Zhang J, Tang H, Li X, et al. Clinical predictors of neurological outcome within $72 \mathrm{~h}$ after traumatic cervical spinal cord injury. Sci Rep. 2016;6:38909.

6. Yuan F, Ding J, Chen H, Guo Y, Wang G, Gao WW, et al. Predicting outcomes after traumatic brain injury: the development and validation of prognostic models based on admission characteristics. J Trauma Acute Care Surg. 2012;73(1):137-45.

7. Diong J, Harvey LA, Kwah LK, Eyles J, Ling MJ, Ben M, et al. Incidence and predictors of contracture after spinal cord injury--a prospective cohort study. Spinal Cord. 2012;50(8):579-84.

8. Sun Y, Chai TC. Role of Purinergic signaling in voiding dysfunction. Curr Bladder Dysfunct Rep. 2010;5(4):219-24.

9. Neirinckx V, Coste C, Franzen R, Gothot A, Rogister B, Wislet S. Neutrophi contribution to spinal cord injury and repair. J Neuroinflammation. 2014;11:150.

10. Lattanzi S, Cagnetti C, Provinciali L, Silvestrini M. Neutrophil-to-lymphocyte ratio predicts the outcome of acute Intracerebral hemorrhage. Stroke. 2016;47(6):1654-7.

11. Wang F, Wang L, Jiang TT, Xia JJ, Xu F, Shen LJ, et al. Neutrophil-to-lymphocyte ratio is an independent predictor of 30-day mortality of Intracerebral hemorrhage patients: a validation cohort study. Neurotox Res. 2018. PMID: 29594812.

12. Zhao JL, Du ZY, Yuan Q, Yu J, Sun YR, Wu X, et al. Prognostic value of neutrophil-to-lymphocyte ratio in predicting the 6-month outcome of patients with traumatic brain injury: a retrospective study. World Neurosurg. 2019. PMID: 30610986

13. Zhao JL, Du ZY, Sun YR, Yuan Q, Yu J, Wu X, et al. Intensive blood pressure control reduces the risk of progressive hemorrhage in patients with acute hypertensive intracerebral hemorrhage: a retrospective observational study. Clin Neurol Neurosurg. 2019;180:1-6.

14. Geisler FH, Coleman WP, Grieco G, Poonian D, Sygen SG. The Sygen multicenter acute spinal cord injury study. Spine (Phila Pa 1976). 2001;26(24 Suppl):S87-98.

15. Bracken MB, Shepard MJ, Collins WF, Holford TR, Young W, Baskin DS, et al. A randomized, controlled trial of methylprednisolone or naloxone in the treatment of acute spinal-cord injury. Results of the second national acute spinal cord injury study. N Engl J Med. 1990;322(20):1405-11.

16. Maas Al, Lingsma HF, Roozenbeek B. Predicting outcome after traumatic brain injury. Handb Clin Neurol. 2015;128:455-74.

17. Yuan Q, Yu J, Wu X, Sun YR, Li ZQ, Du ZY, et al. Prognostic value of coagulation tests for in-hospital mortality in patients with traumatic brain injury. Scand J Trauma Resusc Emerg Med. 2018;26(1):3.

18. Younan D, Lin E, Griffin R, Vanlandingham S, Waters A, Harrigan M, et al. Early trauma-induced coagulopathy is associated with increased ventilatorassociated pneumonia in spinal cord injury patients. Shock. 2016;45(5):502-5.

19. de Campos Guerra JC, Mourao MA, Franca CN, da Rosa CD, Burattini MN. Impact of coagulation in the development of thromboembolic events in patients with spinal cord injury. Spinal Cord. 2014;52(4):327-32.

20. Sun Y, You S, Zhong C, Huang Z, Hu L, Zhang X, et al. Neutrophil to lymphocyte ratio and the hematoma volume and stroke severity in acute intracerebral hemorrhage patients. Am J Emerg Med. 2017:35(3):429-33.

21. You S, Zhong C, Zheng D, Xu J, Zhang X, Liu H, et al. Monocyte to HDL cholesterol ratio is associated with discharge and 3-month outcome in patients with acute intracerebral hemorrhage. J Neurol Sci. 2017;372:157-61.

22. You S, Zhong C, Xu J, Han Q, Zhang X, Liu H, et al. LDL-C/HDL-C ratio and risk of all-cause mortality in patients with intracerebral hemorrhage. Neuro Res. 2016;38(10):903-8

23. Tamhane UU, Aneja S, Montgomery D, Rogers EK, Eagle KA, Gurm HS. Association between admission neutrophil to lymphocyte ratio and outcomes in patients with acute coronary syndrome. Am J Cardiol. 2008;102(6):653-7.

24. Kinoshita K. Traumatic brain injury: pathophysiology for neurocritical care. J Intensive Care. 2016:4:29.

25. Corps KN, Roth TL, McGavern DB. Inflammation and neuroprotection in traumatic brain injury. JAMA Neurol. 2015;72(3):355-62.

26. Lotze MT, Tracey KJ. High-mobility group box 1 protein (HMGB1): nuclear weapon in the immune arsenal. Nat Rev Immunol. 2005;5(4):331-42.

27. Helmy A, De Simoni MG, Guilfoyle MR, Carpenter KL, Hutchinson PJ. Cytokines and innate inflammation in the pathogenesis of human traumatic brain injury. Prog Neurobiol. 2011;95(3):352-72.

28. Shlosberg D, Benifla M, Kaufer D, Friedman A. Blood-brain barrier breakdown as a therapeutic target in traumatic brain injury. Nat Rev Neurol. 2010;6(7):393-403.

\section{Publisher's Note}

Springer Nature remains neutral with regard to jurisdictional claims in published maps and institutional affiliations.
Ready to submit your research? Choose BMC and benefit from:

- fast, convenient online submission

- thorough peer review by experienced researchers in your field

- rapid publication on acceptance

- support for research data, including large and complex data types

- gold Open Access which fosters wider collaboration and increased citations

- maximum visibility for your research: over $100 \mathrm{M}$ website views per year

At BMC, research is always in progress.

Learn more biomedcentral.com/submissions 